\title{
Prognostic value of proliferative activity in lymph node metastases of patients with breast cancer
}

\author{
P J van Diest, E Matze-Cok, J P A Baak
}

\begin{abstract}
Whether the proliferative activity of distant metastases could be predicted by the proliferation in axillary lymph node metastases was investigated in 304 lymph nodes metastases of 52 patients with breast cancer who had not received adjuvant treatment.

The standard deviation of the mean mitotic index (MI)-the average number of mitoses per field in 10 high power fields-was of the best prognostic valve in univariate survival analysis. None of the classic (volume \% epithelium and stroma) or morphometric features (nuclear area, nuclear axis ratio. shape factors) provided significant results. In Cox regression analysis a multivariate combination of the mean MI, the SD of the mean MI, and the maximum MI emerged, which provided a satisfying means of differentiating patients with a good $(68 \%$ survival) and a poor $(28 \%$ survival) prognosis.

Proliferation variables derived from axillary lymph node metastases of patients with breast cancer can predict the clinical course of distant metastases.
\end{abstract}

Lymph node response is the most widely used indication for adjuvant treatment in patients with breast cancer as it is generally recognised to be the best high risk indicator. About $40 \%$ of patients with lymph node metastases, however, are still alive five years after diagnosis, ${ }^{1}$ which indicates that those with positive lymph nodes can be further subdivided into patients with favourable and unfavourable prognoses, respectively.

Several studies have tried to find additional prognostic factors for lymph node positive patients with breast cancer in either the primary tumour or lymph node metastases. The addition of tumour size and the mitotic activity index (MI) of the primary tumour to lymph node response ${ }^{2-7}$ has been shown to improve prognostic prediction in lymph node positive patients. Maehle and Skjaerven reported that the presence of efferent vascular invasion in positive lymph nodes was an unfavourable sign, ${ }^{8}$ and Linden et al showed that a combination of morphometric features from the primary tumour and lymph node metastases had additional prognostic value in lymph node positive patients. ${ }^{9}$

To our knowledge, no study has so far described the prognostic value of proliferation variables derived from lymph node metastases. The aim of this study, therefore, was to evaluate whether proliferative activity of distant breast cancer metastases could be predicted by the degree of proliferation in axillary lymph node metastases compared with classic and morphometric lymph node variables.

\section{Methods}

A total of 52 patients with invasive breast cancer metastatic to the axillary lymph nodes, diagnosed between 1971 and 1981, were included in this study. All patients were treated with radical or modified mastectomy. Postoperative regional radiotherapy was given in all cases, and none of the patients received any form of adjuvant systemic treatment. Mean age at the time of diagnosis was $57 \cdot 3$ years (range 27-84). The median follow up for all patients was 84 months (range 18-215), and the median follow up of the surviving patients was 121 months (range 59-215). Table 1 summarises the clinical data of the patients.

Lymph nodes were identified in the fresh axillary dissection preparation, fixed in neutral buffered formalin, and embedded in paraffin wax. Sections $(4 \mu \mathrm{m}$ thick) were cut and stained with haematoxylin and eosin. All identified lymph nodes were reviewed and all lymph nodes containing metastatic breast cancer cells were further considered for all patients. The total number and percentage of lymph node metastases were registered. Patients had, on average, $6 \cdot 1$ positive lymph nodes (range 1-33). In total, 304 lymph node metastases were thus evaluated.

In all positive lymph nodes of each case the most cellular area containing the highest density of mitotic figures was chosen, excluding areas with necrosis and many non-malignant cells: an area of about $0.5 \times 0.5 \mathrm{~cm}$ was marked. In this area mitotic counting and nuclear measurements were performed.

Table 1 Clinical and typical pathological data

\begin{tabular}{lll}
\hline Age (years) & Mean & $57 \cdot 3$ \\
& Range & $27-84$ \\
Hormonal state & Premenopausal & 22 \\
& Postmenopausal & 30 \\
Tumour size (cm) & Mean & $4 \cdot 0$ \\
& Range & $0 \cdot 8-10 \cdot 0$ \\
Tumour type & Ductal & 44 \\
& Lobular & 8 \\
Number of positive nodes & Mean & $6 \cdot 1$ \\
& Range & $1-33$ \\
Percentage positive nodes & Mean & $42 \cdot 5$ \\
& Range & $4 \cdot 7-100$ \\
\hline
\end{tabular}

ॠOr combinations 
Mitotic figures were counted in 10 consecutive high power fields (HPF) at $\times 400$ magnification using a $\times 40$ objective with an aperture of 0.75 and a field diameter of $450 \mu \mathrm{m}$, starting at the spot in the measurement area with the highest density of mitotic figures. Criteria to identify mitoses have been described elsewhere. ${ }^{10}$ To cope with metastases smaller than $10 \mathrm{HFP}$ the number of mitoses counted was normalised to the average number of mitoses per field (the mitotic index) (MI). All positive lymph nodes of all patients were assessed. The following statistics were calculated for each patient: mean $\mathrm{MI}\left(\mathrm{MI}_{\text {mean }}\right)$; SD of mean MI (SD $\left.M I_{\text {mean }}\right)$; maximum $\mathrm{MI}\left(\mathrm{MI}_{\max }\right)$; minimum $\mathrm{MI}$ $\left(\mathrm{MI}_{\min }\right)$; and $\mathrm{MI}$ range $\left(\mathrm{MI}_{\max }-\mathrm{MI}_{\min }\right)$.

The volume percentages of epithelium (VPE), stroma (VPS), and lumen (VPL) were assessed by point counting on a projection microscope at a magnification of $\times 200$ using a 42 point Weibel grid (line length $15 \mathrm{~mm}$ ). The whole measurement area was considered in each case, regarding only tumour stroma as stroma and lumina as such in cases of clear tubulus formation. The percentages of points falling within each compartment were taken as the volume percentages. In cases of micrometastases, point counting was performed at $\times 400$ magnification to obtain an adequate sample size. A sample size of less than 100 points was considered inadequate, and 15 lymph nodes were rejected from further analysis for this reason. Three more nodes had to be discarded because of the poor quality of the section, leaving 286 lymph nodes. On average, 552 points (range 1021137) were counted for each positive lymph node. The following statistics were calculated for each variable (VPE, VPS, VPL): mean, $\mathrm{SD}$, maximum, minimum and range.

For nuclear morphometry, the lymph node with the highest MI was chosen for each case. Measurements were performed using an interactive digitising video overlay system (PRODIT, Promis Software, Almere, The Netherlands) at a final magnification of $\times 3000$. Nuclei were selected according to the so-called raster method ${ }^{11}$ - that is the systematic sampling of the entire marked area-up to a sample size of 100 or the maximum number of malignant nuclei. For each case, the mean and SD of the nuclear area, nuclear axis ratio, and nuclear form factor were calculated.

For statistical analysis, survival time (defined as the time between data of operation and death from recurrent disease) was used as a follow up variable. Univariate survival analysis was performed according to the Kaplan-Meier method. For continuous variables, cutoffs were

Table 2 Univariate survival analysis data for significant variables

\begin{tabular}{llllll}
\hline Variable & & No of cases & $\%$ Survival & $p$-Value & Mantel-Cox \\
\hline SD MI $_{\text {mean }}$ & $<0.17$ & 12 & 75 & 0.01 & $9 \cdot 1$ \\
& $<0.91$ & 19 & 42 & & \\
MI $_{\text {range }}$ & $\geq 0.91$ & 13 & 23 & 0.02 & 7.6 \\
& $<0.55$ & 15 & 73 & & \\
& $<2.4$ & 13 & 31 & & \\
\hline
\end{tabular}

^Cases with one positive node excluded

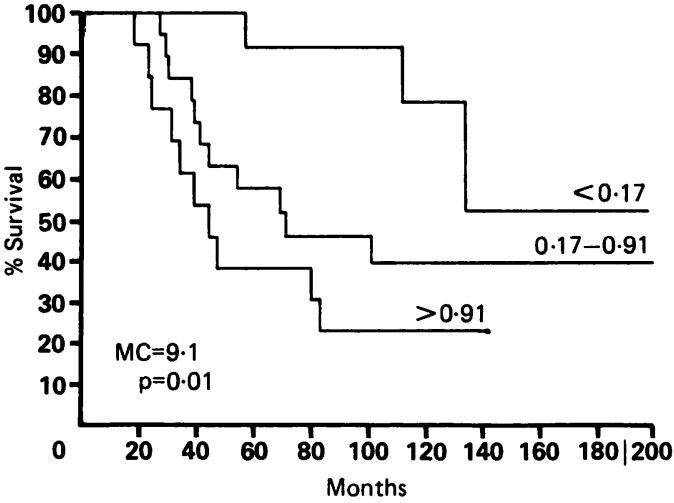

Figure 1 Survival curves for subgroups of lymph node positive patients with different $S D$ values $M I_{\text {mean }}$

chosen in such a way that the patients were divided into two or three groups of roughly the same size. Differences between the curves were analysed using the Mantel-Cox test, with $\mathrm{p}$ values below 0.05 regarded as significant.

Multivariate analysis was performed with the Cox regression model to evaluate the additional prognostic value of the features. All tests were carried out with the BMDP statistical package using the program's life tables (P11) and survival analysis with covariates (P21).

\section{Results}

Of all the statistics taken from the classic and quantitative variables only SD $\mathrm{MI}_{\text {mean }}$ and the $\mathrm{MI}_{\text {range }}$ were significant in univariate survival analysis. Table 2 provides the statistical results for $S D M I_{\text {mean }}$ and $M I_{\text {range }}$. Figure 1 shows the survival curves for subgroups with different values of the $S D \mathrm{MI}_{\text {mean }}$.

In the Cox regression analysis the following multivariate combination of the $\mathrm{MI}_{\text {mean }}, \mathrm{SD}$ $M I_{\text {mean }}$ and maximum $\mathrm{MI}$ emerged:

MVAR $=1.0826^{\star} \mathrm{MI}_{\text {mean }}+2.9998^{\star}$

$\mathrm{SD} \mathrm{MI}$ mean $-1 \cdot 2066^{\star} \mathrm{MI}_{\text {max }}$

This combination was further tested in univariate survival analysis. A cutoff of 0.25 provided a significant differentiation of patients with a good (MVAR of $<0 \cdot 25,68 \%$ survival) and bad (MVAR $\geq 0 \cdot 25,28 \%$ survival) prognosis ( $p=0.0013$, Mantel-Cox 10.4). Figure 2 shows the survival curves for these groups.

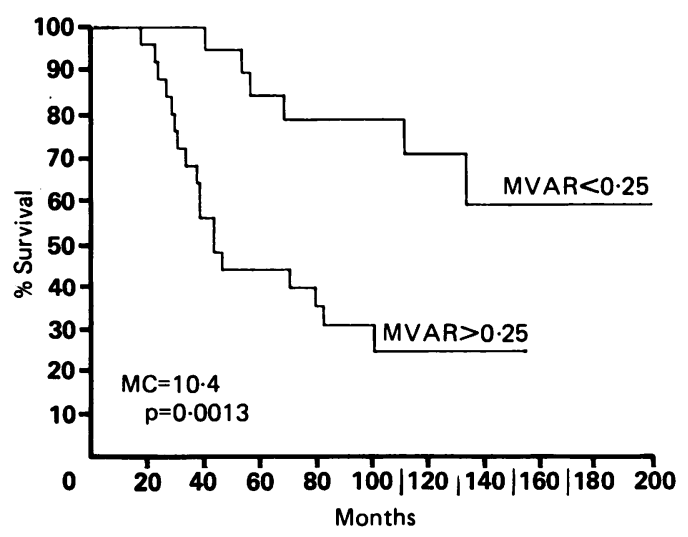

Figure 2 Survival curves for subgroups of lymph node positive patients with different values of $M V A R$. 


\section{Discussion}

Of all the variables evaluated in this study, only the SD of the mean MI and the MI range showed prognostic value in univariate analysis. A multivariate combination of three proliferation variables (mean $\mathrm{SD}$, and maximum lymph node $\mathrm{MI}$ ) adequately discriminated between patients with a relatively favourable and unfavourable prognosis and a long term survival of $68 \%$ and $28 \%$, respectively. It is difficult to find a biological explanation for this multivariate combination, but it may be that a high variation in proliferative activity among different lymph nodes of the same patient indicates a genetic instability that heralds a poor prognosis. Neither the other quantitative variables nor the classic lymph node factors were of any additional prognostic value. Altogether, this points to the intriguing possibility of predicting the behaviour of distant metastases by studying axillary proliferative activity in lymph node metastases of patients with breast cancer.

Linden et al showed that a combination of nuclear axis ratio of the primary tumour and a randomly selected lymph node metastasis had additional prognostic value in lymph node positive patients. ${ }^{9}$ The nuclear axis ratio of the lymph node metastases alone had no prognostic value in this study. Maehle described the prognostic value of mean nuclear area of lymph node metastases, giving no details on what lymph node had been selected. ${ }^{81213}$ In this study the prognostic value of mean nuclear area could not be confirmed. How the prognostic value of lymph node variables derived from this study compare with primary tumour characteristics from a prognostic point of view will be investigated.

An interesting finding from this study was the wide variety in proliferative activity among lymph node metastases in the same patient. One patient with 14 lymph node metastases had an $\mathrm{MI}$ ranging from 0.5 to 9.9 in different positive nodes. This shows that the quantitative phenotype of axillary lymph node metastases from the same patient may be very different. Because tissue processing was stan- dardised and the local circumstances were similar for all axillary lymph nodes, this may indicate that lymph node metastases could be derived from different clones in the primary tumour.

In conclusion, proliferation variables obtained from axillary lymph node metastases of patients with breast cancer have prognostic value and could therefore predict the behaviour of distant metastases in such patients.

The help of Dr J C Fleege in setting up the methodology for the

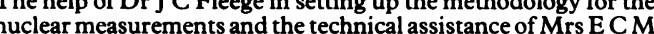
Wisse-Brekelmans is gratefully acknowledged.

This study was financially supported by grant 28-1398 of the Praeventifonds.

1 Nemoto R, Vana J, Bedwani RH, et al. Management and survival of female breast cancer: results of a national survey by the American College of Surgeons. Cancer 1980;45:2917-24.

2 Baak JPA, van Dop H, Kurver PHJ, et al. The value of morphometry to classic prognosticators in breast cancer. Cancer 1985;56:374-82.

3 van Diest PJ, Bak JPA. The morphometric multivariate prognostic index (MPI) is the strongest prognosticator in premenopausal lymph node negative and lymph node positive breast cancer patients. Hum Pathol (in press)

4 van der Linden JC, Baak JPA, Lindeman J, et al. Prospective evaluation of prognostic value of morphometry in patients with primary breast cancer. J Clin Pathol 1987;40:302-6.

5 Uyterlinde AM, Schipper NW, Baak JPA. Comparison of extent of disease, morphometric and DNA flow cytometric prognostic factors in invasive ductal breast cancer. J Clin Pathol 1987;40:1432-6.

6 Uyterlinde AM, Schipper NW, Baak JPA, et al. Limited prognostic value of cellular DNA content to classical and morphometrical parameters in invasive ductal breast cancer. Am J Clin Pathol 1988;89:301-7.

7 Tosi P, Luzi P, Sforza V, et al. Correlation between morphometrical parameters and disease-free survival in morphometrical parameters and disease-free survival in ductai breast can

8 Maehle BO, Skjaerven R. A prognostic index based on the mean nuclear area of breast cancer cells and efferent vascular invasion in the axillary nodes. Diagn Histopathol 1983;6:221-8.

9 van der Linden JC, Baak JPA, Lindeman J, et al. Morphometry and breast cancer. II. Characterisation of breast cancer cells with high malignant potential in patients with spread to lymph nodes: preliminary results. J Clin Pathol 1986;39:603-9.

10 Baak JPA. Counting mitosis. Hum Pathol 1990;21:683-5.

11 Fleege JC, van Diest PJ, Baak JPA. Computer assisted efficiency-testing of different sampling methods for selective nuclear graphic tablet morphometry. Lab Invest tive nuclear
$1990 ; 63: 270-5$.

12 Maehle BO, Thoresen S, Hartveit F. Efferent vascular invasion and the mean nuclear area of axillary nodal tumour cells in breast cancer. Diagn Histopathol 1982;5:297-300.

13 Maehle BO, Thoresen S, Skjaerven R, et al. Mean nuclear area and histological grade of axillary-node tumour in breast cancer, related with prognosis. $\mathrm{Br} J$ Cancer 1982;46:95-100. 